\title{
Measurement of the modulation transfer function (MTF) of a camera lens
}

\section{Aline Vernier, Baptiste Perrin, Thierry Avignon, Jean Augereau, Lionel Jacubowiez}

Aline Vernier, Baptiste Perrin, Thierry Avignon, Jean Augereau, Lionel Jacubowiez, "Measurement of the modulation transfer function (MTF) of a camera lens," Proc. SPIE 9793, Education and Training in Optics and

Photonics: ETOP 2015, 97931 ( (8 October 2015); doi: 10.1117/12.2223114

Event: Education and Training in Optics and Photonics: ETOP 2015, 2015, Bordeaux, France 


\title{
Measurement of the Modulation Transfer Function (MTF) of a camera lens
}

\author{
Aline Vernier, Baptiste Perrin, Thierry Avignon, Jean Augereau, Lionel Jacubowiez \\ Institut d'Optique Graduate School \\ Laboratoire d’Enseignement Expérimental (LEnsE) \\ 2 Avenue Augustin Fresnel, 91127 Palaiseau (France)
}

\begin{abstract}
An accurate characterization of optical elements is one of the pivotal skills to be learnt in the context of undergraduate optics lab work or that of continuing education. We will show an experimental apparatus designed to measure the Modulation Transfer Function (MTF) of a camera lens. The educational scope of this apparatus ranges from the study of the aberrations of the lens and its suitability for use with a given sensor, to the design of the test bench itself.

A first, purely visual observation allows the trainees to single-out the aberrations exhibited by an objective. The presence of the spherical and chromatic aberrations on the axis of the objective is thus identified, and a first estimate of the cut-off frequency of this objective can be given. There, the importance of the geometrical extent of the source and the impact of the optical elements of the test bench on the measurement can also be assessed.

The second part of the experiment makes use of a CMOS camera to measure the Linear Spread Function (LSF) of the camera lens and the MTF is then retrieved by Fourier transform.

The experimental setup consists of a fine slit as a source, a collimator, the studied objective exhibiting conspicuous aberrations and a microscope objective to re-image the LSF either for direct observation with an eye-piece or for analysis with the camera. The latter is performed with a homedeveloped Matlab software.
\end{abstract}

Keywords: Line Spread Function, characterization of optical elements, optical test bench for aberration measurements, Modulation Transfer Function, cut-off frequency and sampling frequency.

Education and Training in Optics and Photonics: ETOP 2015, edited by Eric Cormier, Laurent Sarger Proc. of SPIE Vol. 9793, 97931I - @ 2015 SPIE, IEEE, OSA, ICO · doi: 10.1117/12.2223114 


\section{Scope of the experiment}

This apparatus allows our students to measure the modulation transfer function of a camera lens with $f^{\prime}=\mathbf{2 5 m m}$ and $\mathbf{N}_{\min }=\mathbf{0 . 9 5}$ which is used with a CMOS chip with $5472 \times 3648$ pixels and a pixel size of $2.4 \mu \mathrm{m}$.

Such a delicate measurement is also a good opportunity to demonstrate several key aspects of optical instrumentation, namely, the implementation of a linear source for a given optical system, the impact of the finite size of the source on the measurement, and the choice of the optical elements to image the response of the studied lens on the camera.

The linear source is a practical solution to measure the MTF with light sources which would not be bright enough for use with a point source. The MTF thus measured is in 1D, orthogonally to the direction of the line. This can be proven as follows, given a source $S(x, y)=\delta(x)$. $C$ and a lens of diameter $a$ :

The response of the objective can be expressed as the square of the Fourier transform of the product of the source with the aperture of the lens $R^{2}\left(k_{x}, k_{y}\right)$, with $\left(k_{x}, k_{y}\right)$ the spatial frequencies associated with resp. $(x, y)$.

$$
R\left(k_{x}, k_{y}\right)=\iint_{-a / 2}^{a / 2} e^{i\left(k_{x} x+k_{y} y\right)} \delta(x) \cdot C d x d y
$$

Recalling $\frac{d H(x)}{d x}=\delta(x)$, and integrating by parts yields:

$$
R^{2}\left(k_{x}, k_{y}\right) \propto \frac{\sin ^{2}\left(a k_{y}\right)}{\left(a k_{y}\right)^{2}}
$$

This corresponds to the Linear Spread Function (LSF). The Fourier Transform of the LSF then gives the 1D MTF in the $y$-direction.

\section{Experimental apparatus}

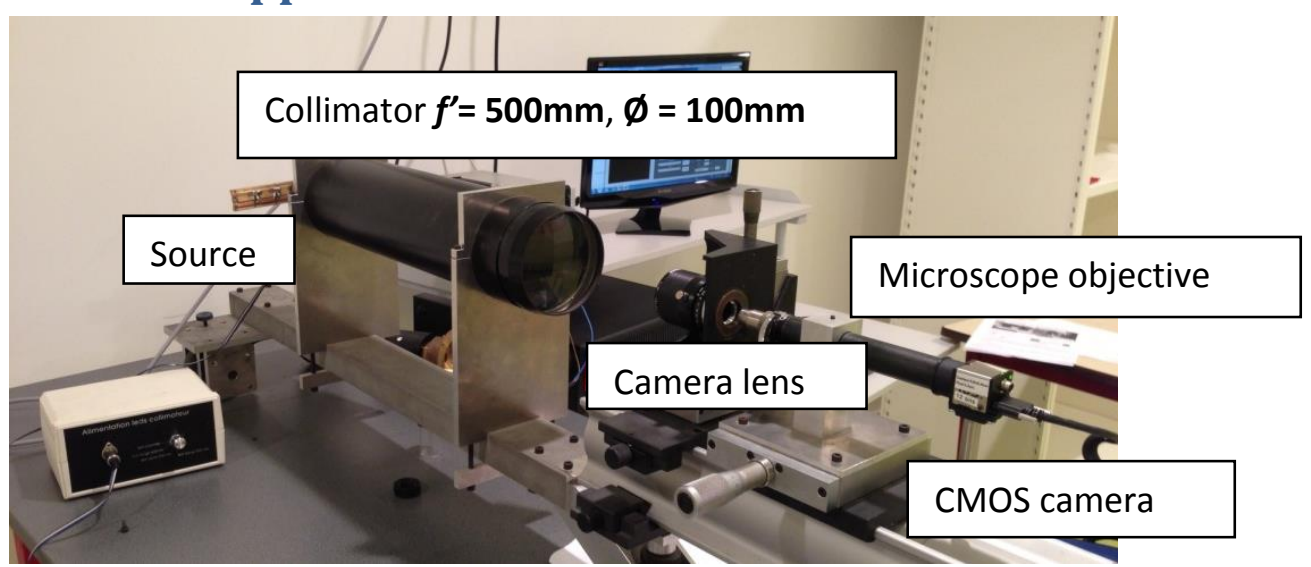

Figure 1: Experimental apparatus picture 


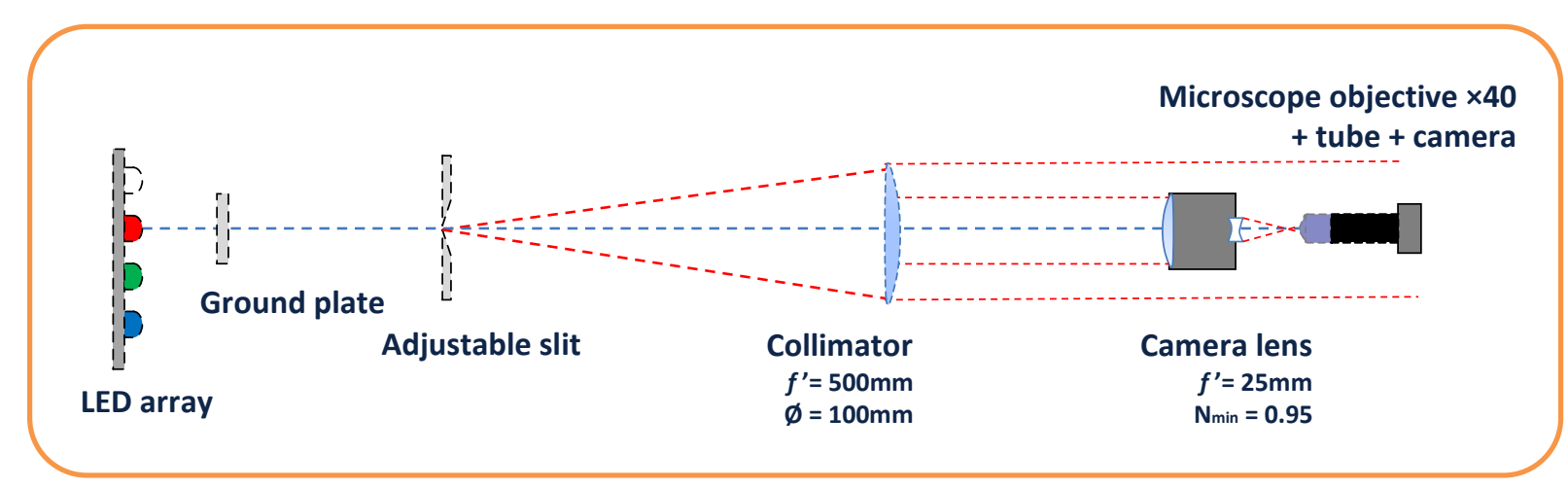

Figure 2: Experimental apparatus schematic

The setup shown on Figure 2 goes as follows:

- Linear source: bright, white or coloured LED light is first diffused by a ground glass diffuser to homogenize the illumination of a thin adjustable slit which acts as a linear source

- Shaping: the source is collimated with a collimator with a focal length $f^{\prime}=500 \mathrm{~mm}$ and a diameter $\emptyset=100 \mathrm{~mm}$

- Camera lens

- Measurement apparatus: a microscope objective x40 re-images the focal spot onto a CMOS chip with $1.3 \times 10^{6}$ square, $5.3 \mu \mathrm{m}$ pixels.

\section{Preliminary considerations}

Prior to diving into the experiment, the students must work out the best theoretical specifications of the studied camera lens: the MTF cut-off frequency $f_{c}$ and the size of the point spread function $\Phi_{\text {Airy }}$.

For $\lambda=630 \mathrm{~nm}, N=0.95$ and $\gamma$ the magnification of the optical system:

$$
\begin{aligned}
& f_{c}=\frac{1}{\lambda N}=1671 \mathrm{~mm}^{-1} \\
& \Phi_{\text {Airy }} \approx 2.44 \lambda N \approx 1.5 \mu \mathrm{m}
\end{aligned}
$$

Because the camera has a pixel size of $2.4 \mu \mathrm{m}$, the system (lens + camera) is thus theoretically limited by the pixel size $\left(f_{c, \text { chip }}=417 \mathrm{~mm}^{-1}\right)$.

From $\Phi_{\text {Airy }}$ they can deduce the maximum size of the point source $d$, given the optical system.

$d \ll \gamma \Phi_{\text {Airy }}$

Given that the focal length of the collimator is $500 \mathrm{~mm}$ and that of the objective is $25 \mathrm{~mm}$, the magnification $\gamma=20$. Therefore, $d \ll 30 \mu \mathrm{m}$ in order to study the camera lens at full aperture.

The students are then asked to work out the sampling spatial frequency of their measurement apparatus to verify that the combination of the $x 40$ microscope objective and the CMOS chip with $1.3 \times 10^{6}$ square, $5.3 \mu$ m pixels is appropriate to measure the MTF of the camera 
lens. A quick calculation yields a sampling frequency of about $7500 \mathrm{~mm}^{-1}$, which is clearly sufficient.

Another important point concerns the choice of the collimator and the microscope objective. It goes without saying that the two must be devoid of aberrations. Moreover, the collimator aperture must be significantly larger than that of the camera lens, and the object numerical aperture of the microscope objective must obviously be greater than the image numerical aperture of the lens not to limit the measurement.

\section{Observations and measurements}

Before making use of the camera and the analysis software, it is informative to make visual observations of the linear spread function. This allows the students to get an intuitive grasp of the aberrations, estimate the size of the LSF and for which aperture size the objective starts to be diffraction-limited.

A more in-depth quantitative analysis is then carried out using the Matlab software provided, the interface of which is shown on Figure 3.

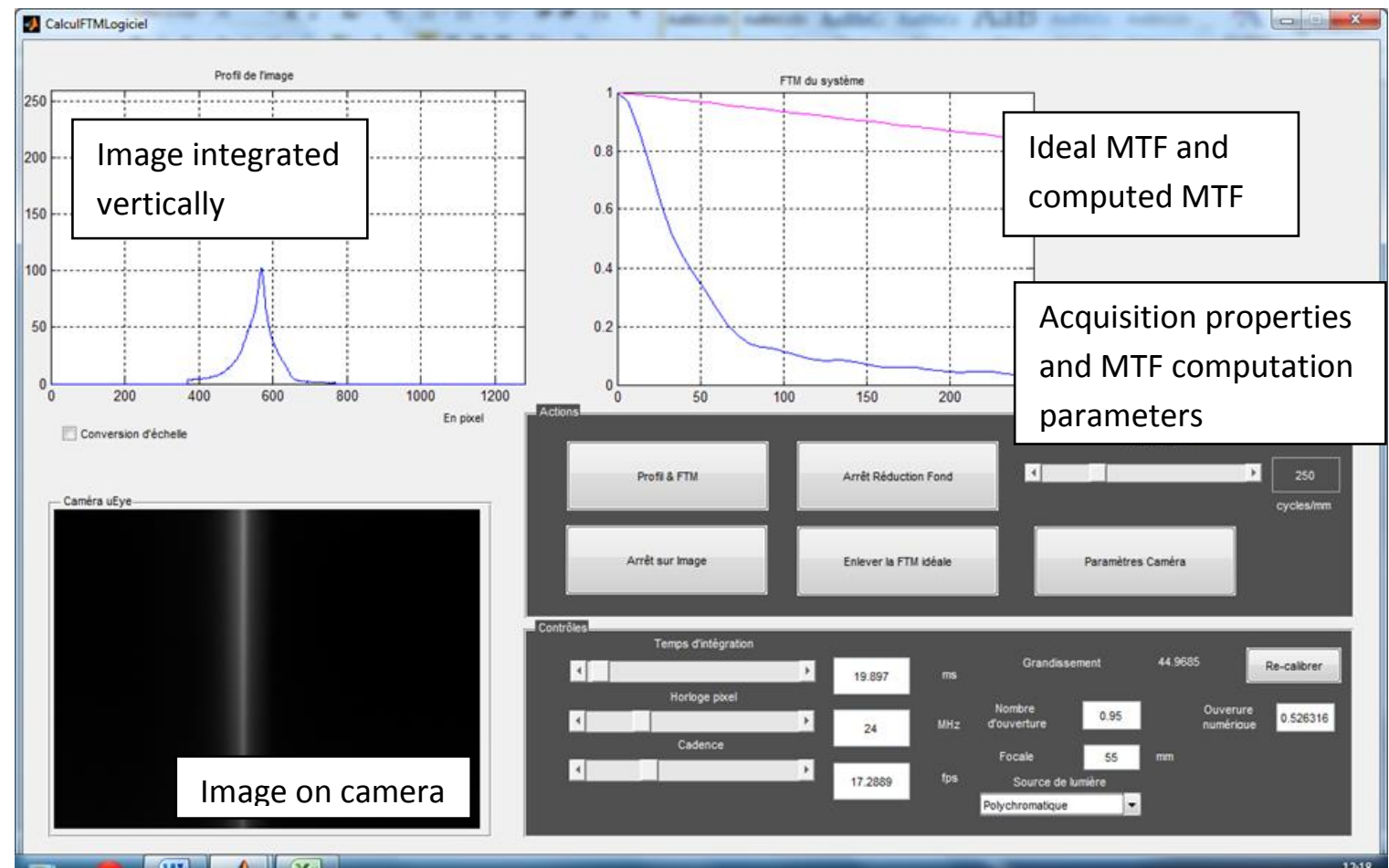

Figure 3: Matlab software interface

After a careful calibration of the magnification of the imaging of the focal spot onto the camera and tuning the exposure time to an appropriate value, the students can view the LSF (e.g. Figure 4) and the measured MTF (e.g. Figure 5) in real time. This allows them to identify the maximum aperture for which the camera lens is diffraction-limited and to quantify the effects of the 
different aberrations, for example the longitudinal displacement of the best focus for different aperture sizes or for different wavelengths, on- and off-axis.

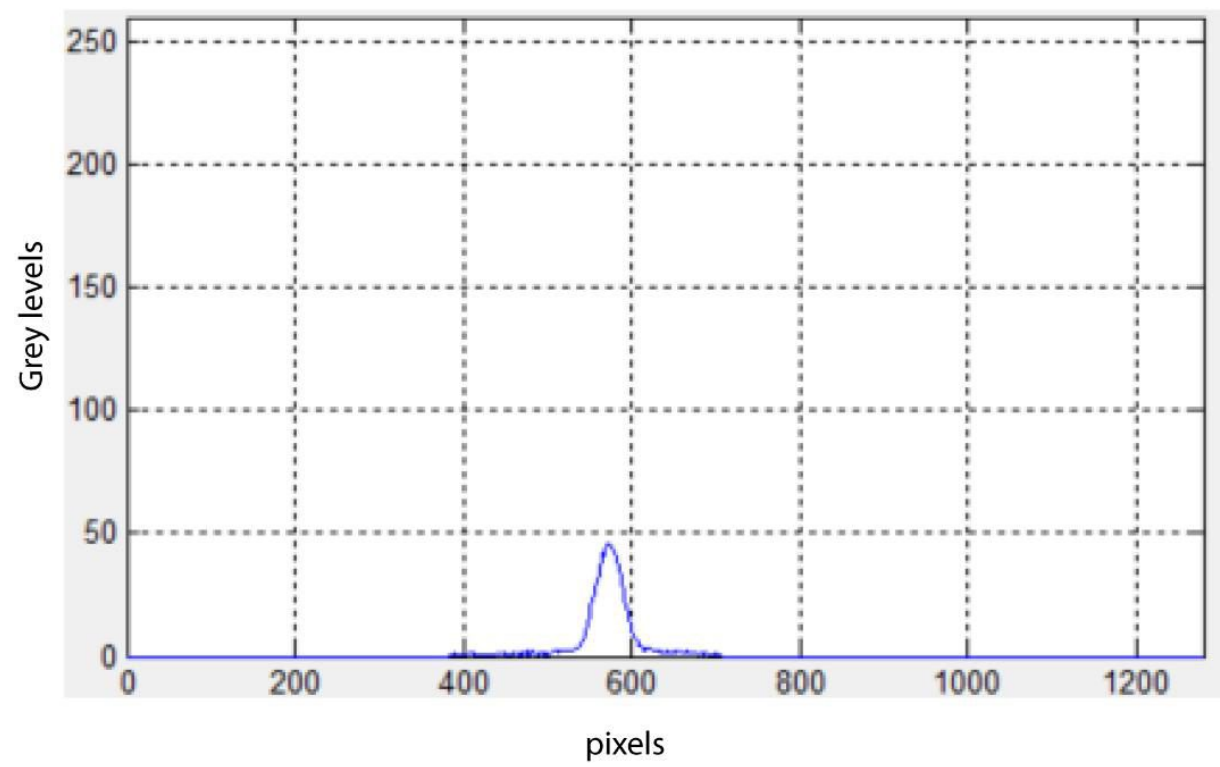

Figure 4: On-Axis Linear Spread Function (integrated vertically from camera image)

$$
\lambda=530 \mathrm{~nm}, N=8
$$

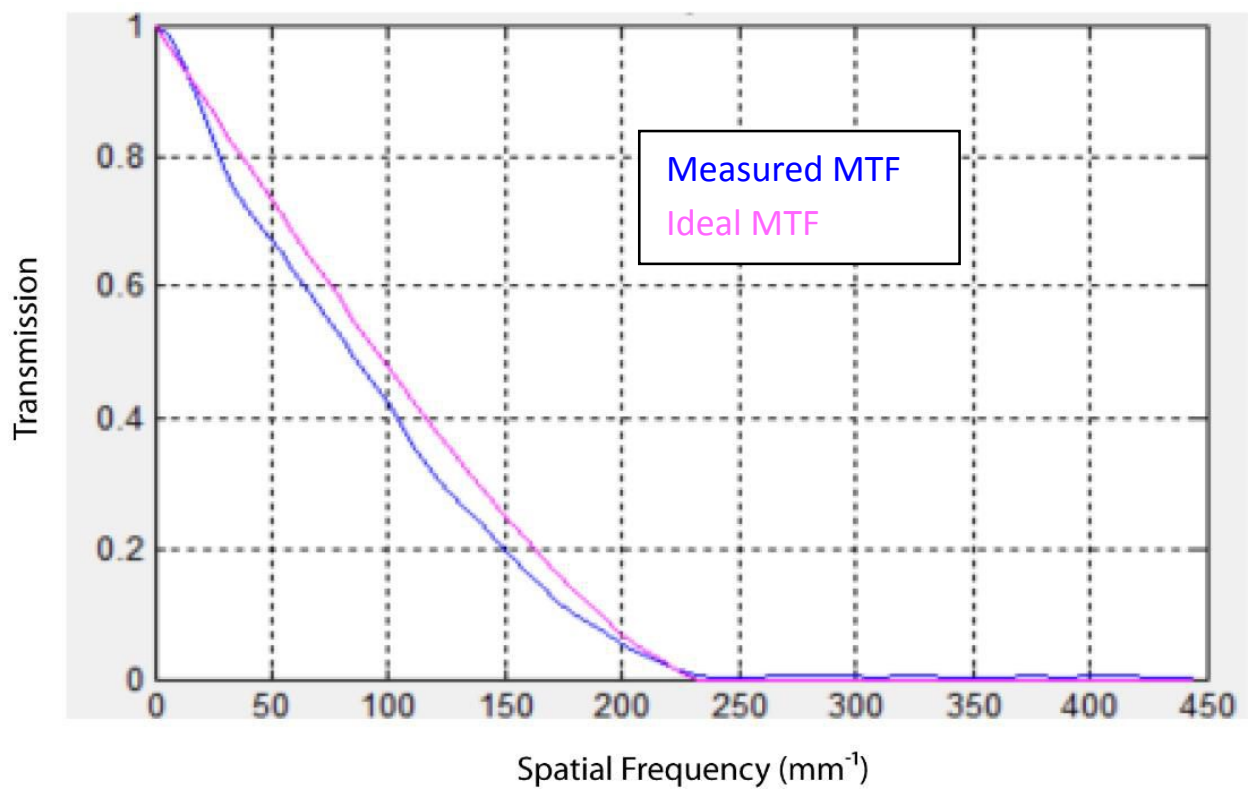

Figure 5: Modulation Transfer Function, $\lambda=530 \mathrm{~nm}, N=8$ 


\section{Conclusion}

We have shown an undergraduate lab setup designed to perform the measurement of the modulation transfer function in 1D of a camera lens. The use of a slit as a linear source allows to work with sources that would not be bright enough for use as a point source. Moreover, the adjustability of the slit width is educationally interesting as the students can directly see the impact of the size of the source on the LSF and subsequently on the measured MTF which they can visualize in real time thanks to a simple GUI. The aberrations of the camera lens can then be studied quantitatively in terms of the MTF. They can then identify that the objective is diffraction-limited from $N=8$. 